\title{
On the $\gamma^{*} \gamma \rightarrow \pi\left(\eta, \eta^{\prime}\right)$ transition form factors
}

\author{
Dmitri Melikhov ${ }^{1,2,3}$ and Berthold Stech ${ }^{4}$ \\ ${ }^{1}$ HEPHY, Austrian Academy of Sciences, Nikolsdorfergasse 18, A-1050 Vienna, Austria \\ ${ }^{2}$ Faculty of Physics, University of Vienna, Boltzmanngasse 5, A-1090 Vienna, Austria \\ ${ }^{3}$ SINP, Moscow State University, 119991 Moscow, Russia \\ 4 ITP, Heidelberg University, Philosophenweg 16, D-69120, Heidelberg, Germany
}

(Dated: July 10, 2018)

\begin{abstract}
The surprising results by the BarBar collaboration on the $\pi \gamma$ transition form factor require new thoughts about the high- $Q^{2}$ dependence of the form factors with virtual photons. We make use of the anomaly sum rule [J. Horejsi and O. Teryaev, Z. Phys. C 65, 691 (1995).] which relates the hadron spectral density to the axial anomaly [S. Adler, Phys. Rev. 177, 2426 (1969); J. S. Bell and R. Jackiw, Nuovo Cimento A 60, 47 (1969).]We study the quark-hadron duality relation for this sum rule and find out that the increase of the rescaled form factor $Q^{2} F_{\pi \gamma}\left(Q^{2}\right) \sim \log \left(Q^{2}\right)$ suggested by the BaBar data requires the presence of a $1 / s$-correction term in the relation between the one-loop spectral density and the hadron-continuum spectral density.

PACS numbers: 11.55.Hx, 12.38.Lg, 03.65.Ge, 14.40.Be
\end{abstract}

\section{MOTIVATION AND RESULTS}

The processes $\gamma^{*} \gamma \rightarrow \pi\left(\eta, \eta^{\prime}\right)$ are of great interest for our understanding of QCD and the meson structure. In particular, the dependence on the space-like momentum $Q$ of the incoming virtual photon has been the subject of detailed experimental [1 $[5]$ and extensive theoretical investigations (recent references see $[6-16])$ ). QCD factorization predicts for large $Q^{2}$ the asymptotic behaviour [17] $Q^{2} F_{\pi \gamma}\left(Q^{2}\right) \rightarrow \sqrt{2} f_{\pi}$, with $f_{\pi}=0.131 \mathrm{GeV}$ denoting the pion decay constant. Similar relations follow for $\eta$ and $\eta^{\prime}$ after taking particle mixing into account. Within errors, this saturation property is indeed observed for the $\eta$ and $\eta^{\prime}$ form factors. However, recent high- $Q^{2}$ data up to $35 \mathrm{GeV}^{2}$ indicate that $Q^{2} F_{\pi \gamma}\left(Q^{2}\right)$ does not saturate at large $Q^{2}$ but increases further. Fig.11 compares these data with the theoretical formula obtained by Brodsky and Lepage [17] which interpolates the values of $F_{\pi \gamma}\left(Q^{2}\right)$ at $Q^{2}=0$ given by the axial anomaly with the asymptotic form mentioned above. So far, no compelling theoretical explanation of the qualitatively different behaviour of the $\pi \gamma$ form factor compared to $\eta \gamma$ and $\eta^{\prime} \gamma$ form factors has been found; as concluded in [7, 11, 13, 14 the behaviour of the $\pi \gamma$ form factor is hard to explain in QCD.

The exact anomaly sum rule [18] offers an interesting possibility [10] to consider the transition form factor without directly referring to QCD factorization theorems. The aim of this letter is to address the $Q^{2}$-behaviour of the $P \gamma$ transition form factor from this perspective.

Our starting point for a treatment of the three processes is the investigation of the amplitude

$$
\begin{array}{r}
\left\langle 0\left|j_{\mu}^{5}\right| \gamma\left(q_{2}\right) \gamma^{*}\left(q_{1}\right)\right\rangle=e^{2} T_{\mu \alpha \beta}\left(p \mid q_{1}, q_{2}\right) \varepsilon_{1}^{\alpha} \varepsilon_{2}^{\beta}, \\
p=q_{1}+q_{2} .
\end{array}
$$

Here $\varepsilon_{1,2}$ denote the photon polarization vectors. This amplitude is considered here for $q_{1}^{2}=-Q^{2}$ and $q_{2}^{2}=0$. Its general decomposition contains four invariant form factors, but for our purpose only the single form factor with the Lorentz structure proportional to $p_{\mu}$ is needed 14]

$$
T_{\mu \alpha \beta}\left(p \mid q_{1}, q_{2}\right)=p_{\mu} \epsilon_{\alpha \beta q_{1} q_{2}} i F\left(p^{2}, Q^{2}\right)+\ldots
$$

The invariant amplitude $F\left(p^{2}, Q^{2}\right)$ may be written in terms of its spectral representations in $p^{2}$ at fixed $Q^{2}$ :

$$
F\left(p^{2}, Q^{2}\right)=\frac{1}{\pi} \int_{4 m^{2}}^{\infty} \frac{d s}{s-p^{2}} \Delta\left(s, Q^{2}\right) .
$$

In perturbation theory one obtains the spectral density as an expansion in powers of $\alpha_{s}$ :

$$
\begin{aligned}
& \Delta_{\mathrm{QCD}}\left(s, Q^{2}\right)= \\
& \quad \Delta_{\mathrm{QCD}}^{(0)}\left(s, Q^{2}\right)+\frac{\alpha_{s}}{\pi} \Delta_{\mathrm{QCD}}^{(1)}\left(s, Q^{2}\right)+O\left(\alpha_{s}^{2}\right),
\end{aligned}
$$

where the lowest order contribution $\Delta_{\mathrm{QCD}}^{(0)}\left(s, Q^{2}\right)$ is obtained from the triangle diagram with the axial current

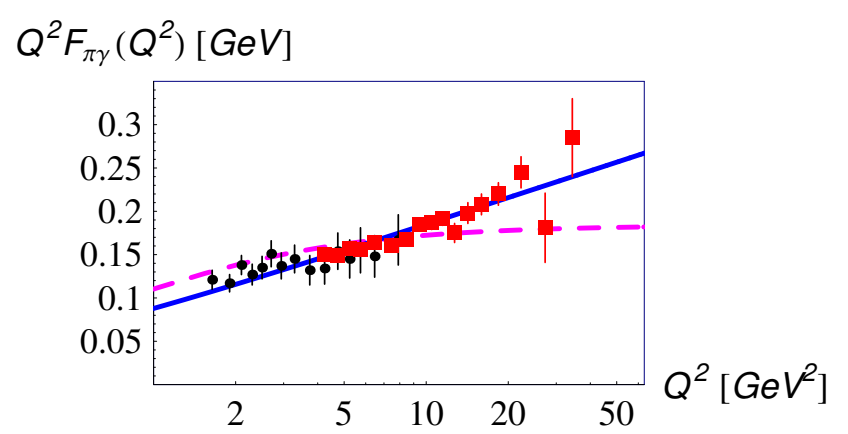

Fig. 1: Form factor $F_{\pi \gamma}$ vs $Q^{2}$ : experimental data from [1, 2] (black dots) and [4] (red squares); dashed line represents the interpolation [17] which coincides with the local-duality model of [14], the solid line represents our fit described below. 
and two vector currents in the vertices [18 20]

$$
\begin{gathered}
\Delta_{\mathrm{QCD}}^{(0)}=\frac{1}{2 \pi} \frac{1}{\left(s+Q^{2}\right)^{2}}\left[Q^{2} w+2 m^{2} \log \left(\frac{1+w}{1-w}\right)\right] \\
w=\sqrt{1-4 m^{2} / s}
\end{gathered}
$$

Here $m$ denotes the mass of the quark propagating in the loop. The integral of $\Delta_{\mathrm{QCD}}^{(0)}\left(s, Q^{2}\right)$ from $s=4 m^{2}$ to infinity has the remarkable property to be independent of $Q^{2}$ and $m[18$ ] and gives the axial anomaly [21]

$$
\int_{4 m^{2}}^{\infty} d s \Delta_{\mathrm{QCD}}^{(0)}\left(s, Q^{2}\right)=\frac{1}{2 \pi}
$$

The Adler-Bardeen theorem [22] states that radiative corrections to the anomaly vanish, requiring that

$$
\int_{4 m^{2}}^{\infty} d s \Delta_{\mathrm{QCD}}^{(i)}\left(s, Q^{2}\right)=0, \quad i \geq 1
$$

This relation however does not require that all higherorder spectral densities $\Delta_{\mathrm{QCD}}^{(i)}\left(s, Q^{2}\right)$ vanish; only the integrals should be zero. The two-loop spectral density $\Delta_{\mathrm{QCD}}^{(1)}\left(s, Q^{2}\right)$ was found to be identically zero [23, 24]. Higher-order spectral densities have not been calculated. However, arguments have been given that all higher-order spectral densities may not vanish identically [14].

Non perturbative QCD interactions will strongly influence $F\left(p^{2}, Q^{2}\right)$ producing a meson pole (and, correspondingly, a (near) delta function of its absorptive part) and a hadron continuum. Nevertheless, the integral over the entire absorptive part $\Delta\left(s, Q^{2}\right)$ remains unchanged. It still represents the anomaly:

$$
\int_{0}^{\infty} d s \Delta\left(s, Q^{2}\right)=\int_{4 m^{2}}^{\infty} d s \Delta_{\mathrm{QCD}}\left(s, Q^{2}\right)=\frac{1}{2 \pi}
$$

For the case of the isovector $\frac{\bar{u} u-\bar{d} d}{\sqrt{2}}$ axial current, the spectrum contains the $\pi^{0}$-meson. Thus, the absorptive part of $F\left(p^{2}, Q^{2}\right)$ has the form

$$
\begin{aligned}
& \Delta\left(s, Q^{2}\right)= \\
& \pi \delta\left(s-m_{\pi}^{2}\right) \sqrt{2} f_{\pi} F_{\pi \gamma}\left(Q^{2}\right)+\theta\left(s-s_{\text {th }}\right) \Delta_{\text {cont }}^{I=1}\left(s, Q^{2}\right) .
\end{aligned}
$$

Here $\Delta_{\text {cont }}^{I=1}\left(s, Q^{2}\right)$ denotes the hadron-continuum contribution in the isovector channel. $F_{\pi \gamma}\left(Q^{2}\right)$ then takes the form

$$
F_{\pi \gamma}\left(Q^{2}\right)=\frac{1}{2 \sqrt{2} \pi^{2} f_{\pi}}\left[1-2 \pi \int_{s_{\mathrm{th}}}^{\infty} d s \Delta_{\mathrm{cont}}^{I=1}\left(s, Q^{2}\right)\right]
$$

For the $\eta \gamma$ and $\eta^{\prime} \gamma$ form factors, one needs to consider the isoscalar currents $\bar{q} q=(\bar{u} u+\bar{d} d) / \sqrt{2}$ and $\bar{s} s$, separately:

$$
\begin{aligned}
& F_{\bar{q} q}\left(Q^{2}\right)=\frac{1}{2 \sqrt{2} \pi^{2} f_{q}}\left[1-2 \pi \int_{s_{\mathrm{th}}}^{\infty} d s \Delta_{\mathrm{cont}}^{I=0}\left(s, Q^{2}\right)\right], \\
& F_{\bar{s} s}\left(Q^{2}\right)=\frac{1}{2 \sqrt{2} \pi^{2} f_{s}}\left[1-2 \pi \int_{s_{\mathrm{th}}}^{\infty} d s \Delta_{\mathrm{cont}}^{\bar{s} s}\left(s, Q^{2}\right)\right] .
\end{aligned}
$$

For each channel, the relevant threshold $s_{\text {th }}$ should be used. Taking $\eta-\eta^{\prime}$ mixing [25, 26] into account and using the corresponding quark charges one finds

$$
\begin{aligned}
F_{\eta \gamma}\left(Q^{2}\right) & =\frac{5}{3 \sqrt{2}} F_{\bar{q} q}\left(Q^{2}\right) \cos \phi-\frac{1}{3} F_{\bar{s} s}\left(Q^{2}\right) \sin \phi \\
F_{\eta^{\prime} \gamma}\left(Q^{2}\right) & =\frac{5}{3 \sqrt{2}} F_{\bar{q} q}\left(Q^{2}\right) \sin \phi+\frac{1}{3} F_{\bar{s} s}\left(Q^{2}\right) \cos \phi .
\end{aligned}
$$

The $\eta-\eta^{\prime}$ mixing angle $\phi$ is known to be $\phi \simeq 39^{\circ}$; the decay constants are taken to be $f_{q}=1.07 f_{\pi}, f_{s}=1.36 f_{\pi}$ [26].

According to (10) and (11), the calculation of the $P \gamma$ form factors requires an Ansatz for the continuum spectral densities $\Delta_{\text {cont }}\left(s, Q^{2}\right)$ for all three cases.

At this place the duality concept may be used by replacing the integrand from threshold to infinity by the integrand obtained from perturbative QCD. For large values of $s$, above the resonance region, the spectral density is anyhow expected to be very well represented by perturbative QCD. At lower $s$, however, the continuum spectral density is strongly distorted by strong interactions and may not be fully accounted for by the rapid onset of the perturbative expression. Moreover, the thresholds should be in general replaced by effective thresholds. This holds in particular for the $\bar{q} q$ and $\bar{s} s$ dispersion representations which determine the $\eta$ and $\eta^{\prime}$ form factors. Below the corresponding effective thresholds, the isoscalar interaction is very weak and is expected to be negligible.

A simple Ansatz for $\Delta\left(s, Q^{2}\right)$ which can to some extent parametrize these effects is to write

$$
\begin{aligned}
& \Delta_{\text {cont }}\left(s, Q^{2}\right)=\theta\left(s-s_{\mathrm{th}}\right) R(s) \Delta_{\mathrm{QCD}}^{(0)}\left(s, Q^{2}\right), \\
& \text { with } R(s)=\left(1-\frac{r}{s}\right) .
\end{aligned}
$$

Finite value of $r, 0 \leq r \leq s_{\mathrm{th}}$, smoothens the threshold behavior. Moreover, the $r / s$ term strongly affects the high- $Q^{2}$ dependence of the form factor. We obtain this way a logarithmic increase with $Q^{2}$ of the form factor multiplied by $Q^{2}$ :

$$
Q^{2} F\left(Q^{2}\right) \sim \frac{Q^{2}}{Q^{2}+s_{t h}}\left(s_{t h}-r\right)+r \log \left(\frac{Q^{2}+s_{t h}}{s_{t h}}\right) .
$$

To derive (14), we set $m=0$ (see the remark below). One may of course assume a more sophisticated behavior in the near-threshold region; in fact, the details of 

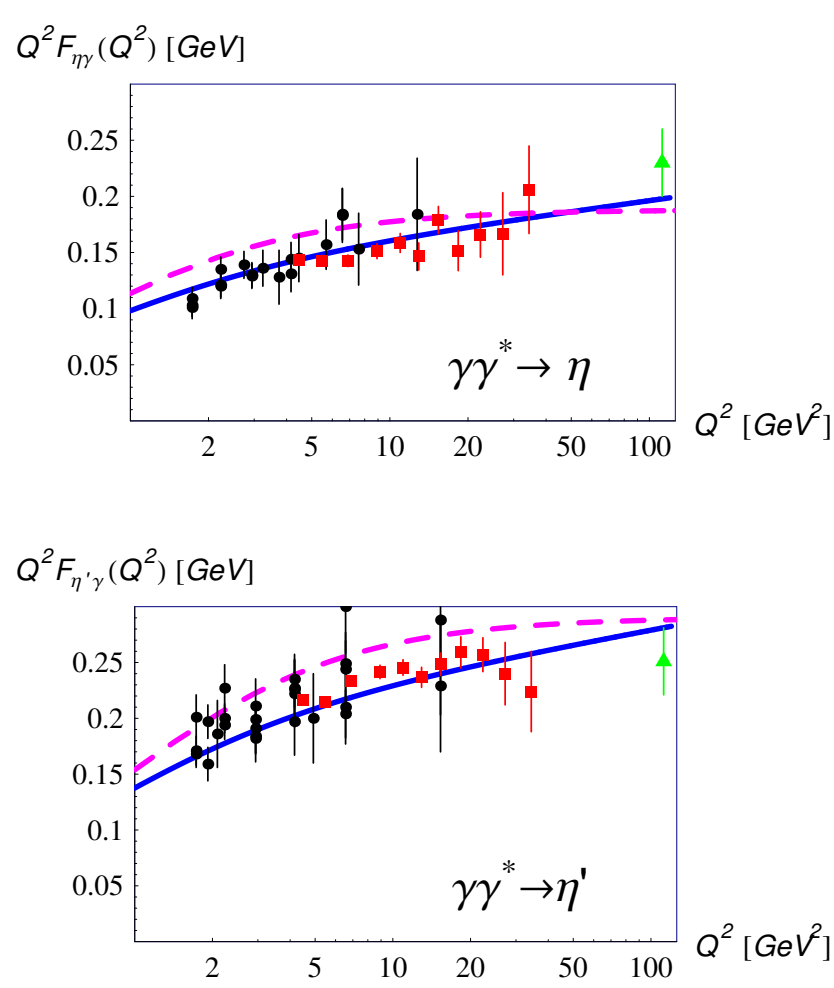

Fig. 2: Form factors $F_{P \gamma}\left(P=\eta, \eta^{\prime}\right)$ vs $Q^{2}$ : experimental data from [1, 2] (black dots), [5] (red squares), and the data borrowed from the time-like region [3] (green triangle). Dashed line - results from local-duality model of [14], solid line - our fit.

$\Delta_{\text {cont }}\left(s, Q^{2}\right)$ at low $s$ influence the form factor at small $Q^{2}$. However, these details do not change the $1 / Q^{2}$ behaviour of $F_{P \gamma\left(Q^{2}\right)}$ at large $Q^{2}$. Our crucial observation is that a wanted $\sim \log \left(Q^{2}\right)$ behavior of $Q^{2} F_{P \gamma}\left(Q^{2}\right)$ requires the presence of the $r / s$ term in $R(s)$ (13).

Making use of the formula (14), one finds values of the fit parameters $s_{\text {th }}$ and $r$ which lead to a good description of all existing experimental data, Figs. 11 and 2. These parameters are as follows: for the pion case we use the physical threshold value $s_{\mathrm{th}}=\left(3 m_{\pi}\right)^{2}=0.165 \mathrm{GeV}^{2}$ and set also $r=\left(3 m_{\pi}\right)^{2}$; then the continuum spectral density vanishes at $s_{\text {th }}$.

It is less obvious which parameter values for the $\eta$ and $\eta^{\prime}$ cases should be used because their structure is influenced by the strong gluon anomaly [26]. We set $s_{\text {th }}=0.56 \mathrm{GeV}^{2}$ for the $\bar{q} q$ channel and $s_{\text {th }}=0.76 \mathrm{GeV}^{2}$ for the $\bar{s} s$ channel. These values are close to the $\{11\}$ and the $\{22\}$ elements of the $\eta-\eta^{\prime}$ mass matrix, respectively [26]. The fit for the $r$ parameters yields $r=0.05$ $\mathrm{GeV}^{2}$ for both channels. A good description of the data is achieved, see Fig. 2. Notice that this value of $r$ is $\sim 1 / 3$ of the corresponding value in the $I=1$ channel. With regard to the quark mass values, one can safely set $m=0$ in (13), at least for the $\pi$-meson case. For the
$F_{\bar{q} q}$ and $F_{\bar{s} s}$ amplitudes needed for the $\eta$ and $\eta^{\prime}$ form factors the dependence on possible values for $m_{q}$ and the strange mass value $m_{s}$ can easily be checked. As long as one sticks to current quark masses there is no visible difference within present accuracies from setting also for these cases $m_{q}=m_{s}=0$. Therefore, the integrals involved above can be calculated analytically. The result has already been shown in (14).

\section{DISCUSSION}

We studied the $\gamma^{*} \gamma \rightarrow P$ transition form factors by means of the exact anomaly sum rule which relates the integral over the hadron spectrum to the axial anomaly. In order to isolate the pseudoscalar mesons from this sum rule, we make use of quark-hadron duality in the following way: we replace the integral over the continuum by the integral over the spectral density given by the diagrams of perturbation theory. We include, howeveressentially, by hand - an additional multiplication factor $R(s)=\left(1-\frac{r}{s}\right)$. This factor goes to unity for high values of $s$ above the resonance region where perturbative QCD should fully describe the continuum. This multiplication factor serves two purposes: (a) It simulates a better threshold behavior and (b) it modifies the high- $Q^{2}$ dependence of the integral over the continuum in a way suggested by recent high- $Q^{2}$ measurements. Our main results are as follows:

(i) The strong difference between the hadron spectral density and the perturbative QCD spectral density in the low- $s$ region influences only the low- $Q^{2}$ structure, but not the large- $Q^{2}$ asymptotics of the form factor obtained from the anomaly sum rule. Using the parameter $r$ different from zero and positive, one easily obtains a good agreement for the low- $Q^{2}$ data, using threshold values well motivated by phenomenology. However, the increase observed for the $\pi \gamma$ form factor by BaBar cannot be understood by subtleties of the behaviour of the continuum spectral density near threshold.

(ii) The deviation of the large- $Q^{2}$ behaviour of the form factor $F_{\pi \gamma}\left(Q^{2}\right)$ from the $1 / Q^{2}$ asymptotics predicted by QCD factorization requires a deviation of order $O(r / s)$ between the hadron-continuum spectral density and the lowest-order perturbative QCD spectral density in the region of larger $s$ values. The multiplication factor $R(s)$ in (13) of the general form

$$
R(s)=1-\frac{r}{s}+\frac{r_{1}}{s^{2}}+\ldots
$$

leads to the form factor which behaves at large $Q^{2}$ as

$$
\begin{aligned}
& Q^{2} F_{P \gamma}\left(Q^{2}\right) \sim r \log \left(Q^{2} / s_{\mathrm{th}}\right) \\
& \quad+\left(s_{\mathrm{th}}-r-r_{1} / s_{\mathrm{th}}\right)+O\left(\log \left(Q^{2}\right) / Q^{2}\right) .
\end{aligned}
$$

The logarithmic increase of $Q^{2} F_{P \gamma}\left(Q^{2}\right)$ occurs proportional to $r$. Terms of higher order of $1 / s$ in $R(s)$ do not 
contribute to the leading $\log \left(Q^{2}\right) / Q^{2}$-behaviour of the form factor.

(iii) The BaBar data on $F_{\pi \gamma}\left(Q^{2}\right)$ are well described by using $s_{\mathrm{th}}=\left(3 m_{\pi}\right)^{2}$, the true threshold for starting the continuum integral, and by setting $r=\left(3 m_{\pi}\right)^{2}$, a constant of the formal order of the light-quark mass. Since a good description of the data is obtained in this way, the theoretical treatment of the underlying $\langle A V V\rangle$ correlator in the chiral limit appears to be insufficient. Further studies of the effects beyond the chiral limit may be needed. Moreover, one should better understand the possible origin of such $O(r / s)$ terms within QCD. So far it is a mere conjecture suggested by the data.

(iv) Obviously, the proposed $O(r / s)$ term in $R(s)$ which modifies the perturbative QCD spectral density leads to the violation of the QCD-factorization theorem. Only if the correction factor $R(s)$ is replaced by unity at some high value of $s$, the form factors multiplied by $Q^{2}$ will saturate at $Q^{2}$ larger than this $s$-value. Since we cannot say where this will occur - if it occurs at all - the true asymptotic behavior for very large $Q^{2}$ cannot be predicted.

Acknowledgments. We are grateful to W. Lucha, J. Pawlowski, and O. Teryaev for valuable discussions. D. M. was supported by the Austrian Science Fund (FWF) under Project No. P22843 and is grateful to the Alexander von Humboldt-Stiftung and the Institute of Theoretical Physics of the Heidelberg University for financial support and hospitality during his stay in Heidelberg.
[1] CELLO Collaboration, H. J. Behrend et al., Z. Phys. C49, 401 (1991)

[2] CLEO Collaboration, J. Gronberg et al., Phys. Rev. D57, 33 (1998).

[3] BaBar Collaboration, B. Aubert et al., Phys. Rev. D74, 012002 (2006).

[4] BaBar Collaboration, B. Aubert et al., Phys. Rev. D80, 052002 (2009).

[5] BABAR Collaboration, P. del Amo Sanchez, Phys. Rev. D84, 052001 (2011).

[6] A. V. Radyushkin, Phys. Rev. D80, 094009 (2009).

[7] H. L. L. Roberts, C. D. Roberts, A. Bashir, L. X. Gutierrez-Guerrero, P. C. Tandy, Phys. Rev. C82, 065202 (2010).

[8] A. Dorokhov, JETP Lett. 91, 163 (2010).

[9] S. S. Agaev, V. M. Braun, N. Offen, and F. A. Porkert, Phys. Rev. D83, 054020 (2011).

[10] Y. N. Klopot, A. G. Oganesian, and O. V. Teryaev, Phys. Lett. B695, 130 (2011); Phys. Rev. D84, 051901(R) (2011).

[11] S. J. Brodsky, F.-G. Cao, and G. F. de Teramond, Phys. Rev. D84, 033001 (2011); Phys. Rev. D84, 075012 (2011).

[12] P. Kroll, Eur. Phys. J. C71 1623 (2011).

[13] A. P. Bakulev, S. V. Mikhailov, A. V. Pimikov, and N. G. Stefanis, Phys. Rev. D84, 034014 (2011); arXiv:1202.1781.
[14] I. Balakireva, W. Lucha, and D. Melikhov, Phys. Rev. D 85, 036006 (2012); arXiv:1103.3781 W. Lucha and D. Melikhov, arXiv:1110.2080.

[15] H. Czyz, S. Ivashyn, A. Korchin, and O. Shekhovtsova, arXiv:1202.1171

[16] C.-C. Lih and C.-Q. Geng, Phys. Rev. C85, 018201 (2012).

[17] G. P. Lepage and S. J. Brodsky, Phys. Rev. D22, 2157 (1980).

[18] J. Horejsi and O. Teryaev, Z. Phys. C65, 691 (1995).

[19] D. Melikhov and B. Stech, Phys. Rev. Lett. 88, 151601 (2002).

[20] D. Melikhov, Eur. Phys. J. direct C4, 2 (2002) arXiv:hep-ph/0110087.

[21] S. Adler, Phys. Rev. 177, 2426 (1969); J. S. Bell and R. Jackiw, Nuovo Cimento 60A, 47 (1969).

[22] S. Adler and B. Bardeen, Phys. Rev. 182, 1517 (1969).

[23] R. S. Pasechnik and O. V. Teryaev, Phys. Rev. D73, 034017 (2006).

[24] F. Jegerlehner and O. V. Tarasov, Phys. Lett. B639, 299 (2006).

[25] V. V. Anisovich, D. I. Melikhov, and V. A. Nikonov, Phys. Rev. D55, 2918 (1997).

[26] T. Feldmann, P. Kroll, and B. Stech, Phys. Rev. D58, 114006 (1998); Phys. Lett. B449, 339 (1999). 